\title{
Satellite Clock Error and Orbital Solution Error Estimation for Precise Navigation Applications
}

\author{
Bharati Bidikar ${ }^{1}$, Gottapu Sasibhushana Rao ${ }^{2}$, Laveti Ganesh ${ }^{3}$, MNVS Santosh Kumar ${ }^{4}$ \\ ${ }^{1}$ Department of ECE, Andhra University, Visakhapatnam, India; ${ }^{2}$ Department of ECE, Andhra University, Visakhapatnam, India; \\ ${ }^{3}$ Department of ECE, ANITS, Visakhapatnam, India; ${ }^{4}$ Department of ECE, AITAM, Tekkali, India. \\ Email: Bharati.Bidikar@gmail.com
}

Received November $5^{\text {th }}, 2013$; revised December $5^{\text {th }}, 2013$; accepted December $12^{\text {th }}, 2013$

Copyright (C) 2014 Bharati Bidikar et al. This is an open access article distributed under the Creative Commons Attribution License, which permits unrestricted use, distribution, and reproduction in any medium, provided the original work is properly cited. In accordance of the Creative Commons Attribution License all Copyrights (C) 2014 are reserved for SCIRP and the owner of the intellectual property Bharati Bidikar et al. All Copyright (C) 2014 are guarded by law and by SCIRP as a guardian.

\section{ABSTRACT}

Global Positioning System (GPS) is a satellite-based navigation system that provides a three-dimensional user position $(x, y, z)$, velocity and time anywhere on or above the earth surface. The satellite-based position accuracy is affected by several factors such as satellite clock error, propagation path delays and receiver noise due to which the GPS does not meet the requirements of critical navigation applications such as missile navigation and category I/II/III aircraft landings. This paper emphasizes on modelling the satellite clock error and orbital solution (satellite position) error considering the signal emission time. The transmission time sent by each satellite in broadcast ephemerides is not accurate. This has to be corrected in order to obtain correct satellite position and in turn a precise receiver position. Signal transmission time or broadcast time from satellite antenna phase center is computed at the receiver using several parameters such as signal reception time, propagation time, pseudorange observed and satellite clock error correction parameters. This corrected time of transmission and broadcast orbital parameters are used for estimation of the orbital solution. The estimated orbital solution was validated with the precise ephemerides which are estimated by Jet Propulsion Laboratory (JPL), USA. The errors are estimated for a typical day data collected on $11^{\text {th }}$ March 2011 from dual frequency GPS receiver located at Department of Electronics and Communication Engineering, Andhra University College of Engineering, Visakhapatnam $\left(17.73^{\circ} \mathrm{N} / 83.319^{\circ} \mathrm{E}\right)$.

\section{KEYWORDS}

\section{Satellite Clock Error; Satellite Clock Offset; Orbital Solution; Broadcast Ephemerides}

\section{Introduction}

GPS has been widely used for precise positioning and navigation applications. In addition to the propagation errors, the receiver position accuracy, availability, reliability and integrity of GPS navigation solution are affected by satellite clock errors and orbital solution errors. Individual satellite clocks, although highly stable, may deviate from GPS system time. Hence for precise navigation applications, satellite clock error needs to be corrected.

In this paper, the satellite signal time of transmission is precisely estimated by considering the clock correction parameters like satellite clock bias $\left(\mathrm{a}_{0}\right)$, satellite clock drift $\left(\mathrm{a}_{1}\right)$, satellite clock drift rate $\left(\mathrm{a}_{2}\right)$, transmitted as part of the navigation message, signal emission time at the antenna and the pseudorange measured between the satellite and the receiver.

The orbital solution of each satellite is estimated using the broadcast ephemerides, which differ from their true satellite positions as shown in Figure 1. This deviation is due to uncertainty in the gravitational model, limited accuracy of the orbit representations and inadequately modelled surface forces on the satellites like solar radiation pressure and atmospheric drag. In this paper, the orbital solution errors are estimated by comparing the broadcast ephemerides with the precise ephemerides obtained by JPL, USA. Here for error calculation, the JPL estimations are taken as the benchmark assuming that these estimates 


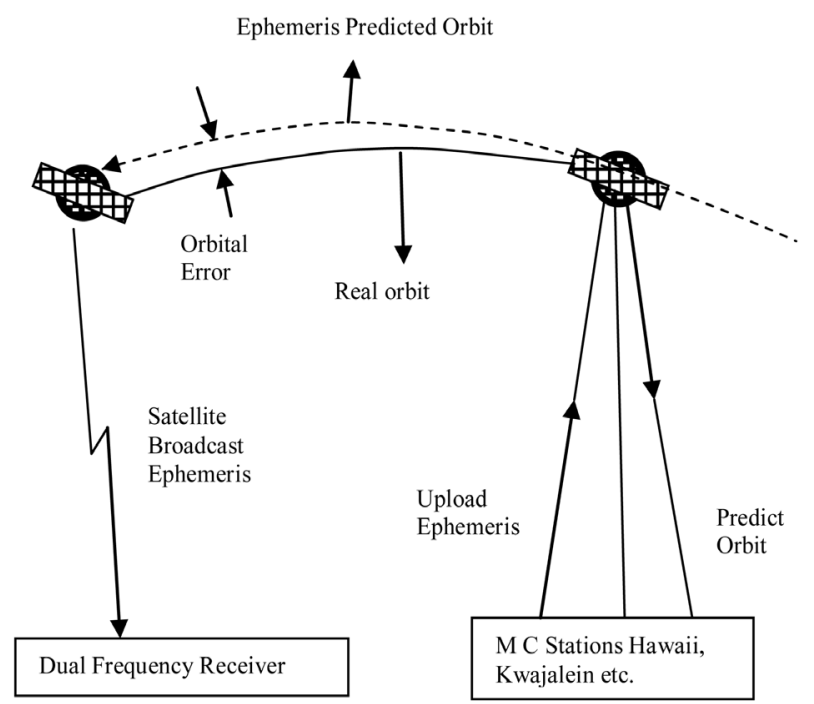

Figure 1. Satellite ephemeris errors.

are of high precision. The errors are estimated and analysed for ephemeris data collected on $11^{\text {th }}$ March 2011 at Department of Electronics and Communication Engineering, Andhra University College of Engineering, Vishakhapatnam.

\section{Estimation of Satellite Clock Error and Orbital Solution Error}

The GPS receiver uses the same PRN codes which are transmitted by the 32 satellites for determining the distance between each satellite and receiver. This distance is also known as pseudorange. For precise navigation solution the computed pseudorange needs to be corrected for the errors like satellite clock error, tropospheric error, multipath errors, ionospheric errors etc. [1]. Among these the satellite clock error has a major impact on the pseudorange and is given by the following Equation (1)

$$
P_{m}=\rho+\varepsilon^{s c} \times C
$$

where, $P_{m}=$ Measured range (meters); $\rho=$ True range (meters); $\varepsilon^{s c}=$ Satellite clock error (sec); $C=$ Velocity of light $=3 \times 10^{8}$ meters $/ \mathrm{sec}$; from Equation (1) it is evident that satellite clock error of 1 microsecond will lead to 300 meters error in the pseudorange [2].

\subsection{Satellite Clock Error}

All satellites contain atomic clocks that control all onboard timing operations, including broadcast signal generation. Although, these clocks are highly stable still they lack of perfect synchronization between the timing of the satellite broadcast signals and GPS system time. Satellite clock correction terms $\left(a_{0}, a_{1}\right.$ and $\left.a_{2}\right)$ and time of clock $t_{o c}$, account for this lack of synchronization. All these parameters are obtained from navigation file. The on board clock stability is about 1 to 2 parts in $10^{13}$ over a period of one day [3]. These errors are common to all users observing the same satellite. The satellite clock error is caused by the satellite oscillator not being synchronised to true time (GPS time).

The deviation of a particular clock from GPS system time is modeled as a quadratic function of time. The parameters of this model are estimated, uploaded to the satellite, and are broadcasted. The coefficients $a_{0}, a_{1}$ and $a_{2}$ in the below equation are called the bias, drift, and aging parameters of the satellite clock [4]. The estimation of satellite clock errors are as given below.

The satellite time of transmission is computed as

$$
t_{s}=t_{r x}-(P / c)
$$

where, $t_{s}=$ satellite time of transmission (sec); $t_{r x}=$ Signal reception time at receiver (sec); $P=$ Pseudorange (meters); $c=$ speed of light (meters/sec).

The time at which the signal received from the satellite and the pseudorange are read from the broadcast ephemerides and observation data files respectively. The term $(P / c)$ in Equation (2) gives the signal propagation time. This propagation time is subtracted from the signal reception time computed at the receiver and the residual time will be the instant at which the signal started from the satellite.

The satellite clock offset $(d t)$ is calculated as,

$$
d t=t_{s}-t_{o c}
$$

where, $t_{s}=$ Satellite time of transmission (sec); $t_{o c}=$ Time of clock (sec).

The satellite time of transmission is calculated using Equation (2) and time of clock is available in navigation data.

The satellite clock error $\left(\varepsilon^{s c}\right)$ is modelled using the second order polynomial as given below. The correction parameters bias, drift and aging are available in navigation data.

$$
\varepsilon^{s c}=a_{0}+a_{1} d t+a_{2} d t^{2}
$$

where, $a_{0}=$ clock bias (sec); $a_{1}=$ clock drift (sec/sec); $a_{2}=$ frequency drift (i.e. aging) (sec/sec $\left.{ }^{2}\right) ; d t=$ satellite clock offset (sec).

The GPS time of transmission corresponds to the signal emission time from the satellite and is computed as below,

$$
T_{G P S}=t_{s}-\varepsilon^{s c}
$$

where, $T_{G P S}=$ Corrected GPS time of transmission (sec); $t_{s}=$ satellite time of transmission (sec); $\varepsilon^{s c}=$ Satellite clock error (sec).

Finally the satellite position is estimated using the corrected time of transmission $T_{G P S}$ and the broadcast orbital parameters. 


\subsection{Broadcast Orbital Solution Error}

The GPS navigation message file which contains the broadcast ephemerides gives the Keplerian parameters needed to compute the coordinates and clock correction for each satellite. This ephemeris data also contains the time information needed to correct that satellite's clock to match with the GPS time. Error in satellite clock and orbital parameters results in estimation of incorrect orbital solution.

\subsection{JPL Orbital Solution Interpolation}

The Lagrange interpolation algorithm is used to find the intermediate values for the points (known as the data nodes) at a given interval. The precise satellite positions estimated by JPL for an epoch interval of 15 minutes where as broadcast satellite positions are estimated for an epoch interval of 15 seconds. The precise 3D satellite positions, $S_{k}$ at different regular intervals of time are used to interpolate the satellite positions in between the known intervals by using the Lagrange interpolation algorithm [5],

$$
P(x)=y_{1} L_{1}(x)+y_{2} L_{2}(x)+\cdots+y_{n} L_{n}(x)
$$

where, $L_{1}(x), L_{2}(x), \cdots, L_{n}(x)$ are Lagrange interpolating polynomials defined as,

$$
L_{i}(x)=\frac{\prod_{j=1}^{n}\left(x-x_{j}\right) \quad j \neq i}{\prod_{j=1}^{n}\left(x_{i}-x_{j}\right) \quad j \neq i} i=1,2,3, \cdots, n
$$

where, $x=$ Lagrange multipliers; $n=$ Number of intervals.

The above Lagrange interpolating polynomial for time interval $t$ is written as the $\mathrm{n}^{\text {th }}$ polynomial

$$
L_{n}(t)=\frac{\left(t-t_{1}\right)\left(t-t_{2}\right)\left(t-t_{3}\right) \cdots\left(t-t_{n-1}\right)}{\left(t_{n}-t_{1}\right)\left(t_{n}-t_{2}\right)\left(t_{n}-t_{3}\right) \cdots\left(t_{n}-t_{n-1}\right)}
$$

These calculated polynomials are substituted in Equation (6) to get the interpolated precise satellite positions.

\section{Results and Discussion}

The satellite clock errors and orbital solution errors are estimated and the analysis of the error which are supported by the relevant graphs and the tables are presented in this paper.

The broadcast orbital solution error and satellite clock error are estimated using the data collected by the receiver from 02:00 hours to 02:39 hours with an epoch interval of 15 seconds. During this observation period of 39 minutes (total of 157 epochs) out of 31 satellites, 9 (02, $05,12,15,18,21,25,26,29)$ satellites were visible. Though the errors are computed and analyzed for all the visible satellites, the results corresponding to a particular Satellite Vehicle Pseudo Random Noise (SVPRN 26) are presented in this paper. To compute the positional error of the satellite, the positions estimated by the receiver are compared with the one estimated by JPL, assuming the JPL estimates are of high precision.The precise ephemerides estimated by JPL are interpolated using the Lagrange interpolation algorithm to generate satellite position for SVPRN 26 for 157 epochs with an epoch interval of 15 seconds. Tables given in this paper details 10 epochs of data and figures are plotted for 157 epochs.

The broadcast ephemerides are collected on $11^{\text {th }}$ March 2011 from the dual frequency GPS receivers located at Department of Electronics and Communication, Andhra University College of Engineering, Visakhapatnam $\left(17.73^{\circ} \mathrm{N} /\right.$ $83.319^{\circ} \mathrm{E}$ ). The precise ephemerides are collected from Jet Propulsion Laboratory (JPL), USA for the same day.

\subsection{Satellite Clock Error}

Table 1 details the satellite clock offset and satellite clock error of SVPRN26 for 10 epochs. Figure 2 and Figure 3 shows these variations over 157 epoch and it is observed that the satellite clock offset is varied from -0.07262 to 2340 seconds and the corresponding clock error is varied from -0.00014192 to -0.00014194 seconds.

\subsection{Orbital Solution Error}

Table 2 details the error in each of the coordinates of the broadcast satellite position with that of the precise satellite position of SVPRN26 for 10 epochs. Figure 4 shows this variation over 157 epochs and it is observed that the satellite positions in the $\mathrm{x}$-coordinate is deviated by 17.02 to -5.939 meters. Similarly, the error is varied from 2.973 to 63.89 meters and -235.8 to -218.1 meters for $y-$ and z-coordinates respectively.

Table 1. Satellite clock offset and error of SVPRN 26.

\begin{tabular}{cccc}
\hline Epoch No. & $\begin{array}{c}\text { Time of epoch } \\
\text { (sec) }\end{array}$ & $\begin{array}{c}\text { Satellite clock } \\
\text { offset (sec) }\end{array}$ & $\begin{array}{c}\text { Satellite clock } \\
\text { error (sec) }\end{array}$ \\
\hline 1 & 439200 & -0.07262 & -0.00014192 \\
2 & 439215 & 14.9274 & -0.00014192 \\
3 & 439230 & 29.92741 & -0.00014192 \\
4 & 439245 & 44.92743 & -0.00014192 \\
5 & 439260 & 59.92745 & -0.00014192 \\
6 & 439275 & 74.92746 & -0.00014192 \\
7 & 439290 & 89.92748 & -0.00014192 \\
8 & 439305 & 104.9275 & -0.00014192 \\
9 & 439320 & 119.9275 & -0.00014192 \\
10 & 439335 & 134.9275 & -0.00014192 \\
\hline
\end{tabular}




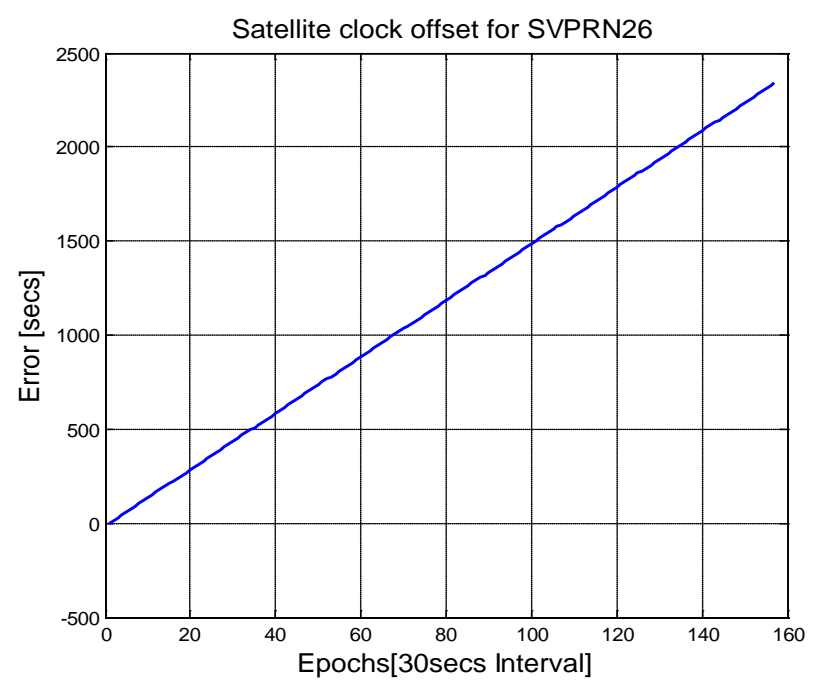

Figure 2. Satellite clock offset for SVPRN 26.

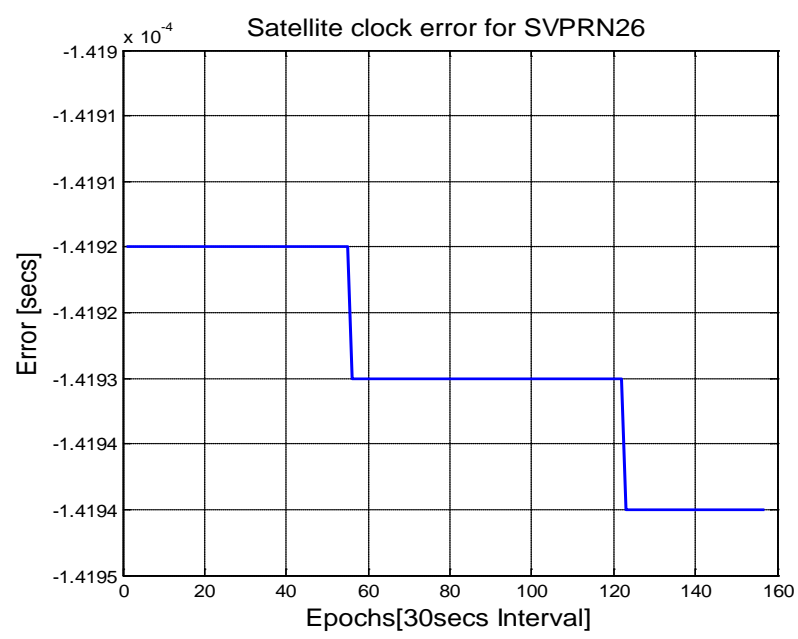

Figure 3. Satellite clock error for SVPRN26.

Table 2. Orbital solution error for SVPRN 26.

\begin{tabular}{ccccc}
\hline Epoch No. & $\begin{array}{c}\text { Time of } \\
\text { epoch(s) }\end{array}$ & \multicolumn{3}{c}{$\begin{array}{c}\text { Orbital solution error } \\
\text { (X,Y,Z) SVPRN 26 (m) }\end{array}$} \\
\hline 1 & 439200 & 17.018 & 2.973 & -235.762 \\
2 & 439215 & 16.799 & 3.351 & -235.716 \\
3 & 439230 & 16.581 & 3.729 & -235.670 \\
4 & 439245 & 16.363 & 4.107 & -235.623 \\
5 & 439260 & 16.147 & 4.486 & -235.574 \\
6 & 439275 & 15.931 & 4.864 & -235.525 \\
7 & 439290 & 15.716 & 5.243 & -235.475 \\
8 & 439305 & 15.503 & 5.623 & -235.424 \\
9 & 439320 & 15.290 & 6.002 & -235.372 \\
10 & 439335 & 15.078 & 6.382 & -235.320 \\
\hline
\end{tabular}

\section{Conclusions}

This paper reveals the importance of the satellite clock error and the orbital solution error.

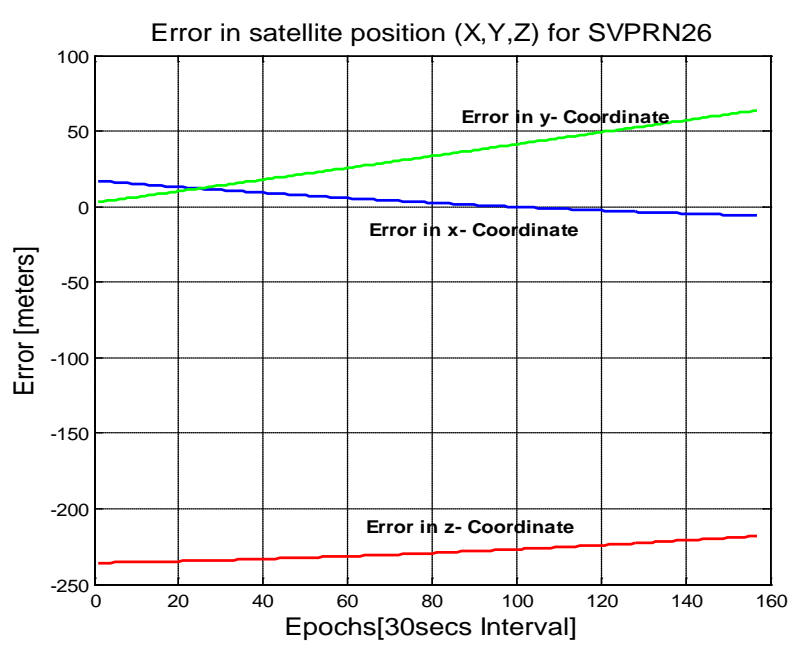

Figure 4. Orbital solution error for SVPRN 26.

Satellite clock error. Over 157 epochs of data analysed, it is observed that the satellite clock offset varied from $-\mathbf{0 . 0 7 2 6 2}$ to 2340 seconds and the corresponding clock error varied from $\mathbf{- 0 . 0 0 0 1 4 1 9 2}$ to $\mathbf{- 0 . 0 0 0 1 4 1 9 4}$ seconds. Hence the clock error estimation needs to be modeled for precision applications, (e.g. CAT I/II aircraft landings, missile navigation).

\section{Broadcast Orbital Solution Errors.}

The error in satellite positions inturn affects the accuracy of navigation solution. Broadcast satellite position varied from precise positions and the deviation in $\mathrm{x}$-coordinate is $\mathbf{1 7 . 0 2}$ to $\mathbf{- 5 . 9 3 9}$ meters over 157 epochs. Similarly the error varied from 2.973 to $\mathbf{6 3 . 8 9}$ meters and $-\mathbf{2 3 5 . 8}$ to $-\mathbf{2 1 8 . 1}$ meters for $\mathrm{y}$ - and z-coordinates respectively. These positional errors have significant effect on the critical applications, (e.g. studies of the crustal dynamics of the earth).

\section{Acknowledgements}

The work undertaken in this paper is supported by Ministry of Science and Technology, Department of Science and Technology (DST), Government of India, New Delhi, under Woman Scientist Scheme(WOS-A), Vide Saction letter No. SR/WOS-A/ET-04/2013.

\section{REFERENCES}

[1] M. Pratap and E. Per, “Global Positioning System: Signals, Measurements and Performance,” 2nd Edition, Ganga-Jamuna Press, New York, 2006.

[2] E. L. Akim and D. A. Tuchin, "GPS Errors Statistical Analysis for Ground Receiver Measurements,” Keldysh Institute of Applied Mathematics, Russia Academy of Sciences, 2002.

[3] G. S. Rao, “Global Navigation Satellite Systems,” Mc 
Graw-Hill Education, New Delhi, 2010.

[4] E. D. Kaplan, "Understanding GPS: Principles and Applications,” 2nd Edition, Artech House Publishers, Boston,
2006.

[5] K. Borre and G. Strang, "Linear Algebra Geodesy and GPS,” Wellesley-Cambridge Press, Cambridge, 1997. 